\title{
Holistic nursing care: theories and perspectives
}

\author{
Ioanna Papathanasiou ${ }^{1}$, Melachrini Sklavou ${ }^{2}$, Lambrini Kourkouta $^{3}$ \\ ${ }^{1}$ Nursing Department, Technological Educational Institute of Larissa, Greece \\ ${ }^{2}$ General Hospital of Larissa, Greece \\ ${ }^{3}$ Nursing Department, Technological Educational Institute of Thessaloniki, Greece
}

Email address:

iopapathanasiou@yahoo.gr (I. Papathanasiou), papathan@teilar.gr (I. Papathanasiou)

To cite this article:

Ioanna Papathanasiou, Melachrini Sklavou, Lambrini Kourkouta. Holistic Nursing Care: Theories and Perspectives. American Journal of Nursing Science. Vol. 2, No. 1, 2013, pp. 1-5. doi: 10.11648/j.ajns.20130201.11

\begin{abstract}
Introduction: Holistic medicine and holistic nursing aim at the improvement of healing of the individual as a bio-psycho-social unity, from birth until death. Aim: To search for current data on the value and applications of holistic nursing, placing emphasis on the field of psychiatry. Literature review: International data bases and internet were searched (including Pubmed/Medline). Emphasis was placed on theoretical background, on alternative therapies and on the practical applications of holistic care. Conclusion: Holistic approach takes into account the biological, social and spiritual needs of the patient. A considerable number of alternative therapies can contribute to the management of psychiatric and other diseases.
\end{abstract}

Keywords: Holistic Care; Nursing; Psychiatry; Alternative Therapies

\section{Introduction}

The technological advancement in the field of biology and the over-specialization of the health personnel has led to the successful treatment of previously considered incurable conditions. However, the fact that several diseases have been treated with success does not necessarily guarantee the long-term rehabilitation of the patient. Health is a complex concept, which refers mainly to an individual's well-being in a given environment, rather than to the absence of a disease, as that is defined by pathology and the traditional biomedical health approach. Moreover, the symptoms of a disease reflect a deeper disturbance of the biopsychosocial balance of an individual and thusly, the definitive treatment of the "disease" lies mainly on treating effectively the underlying causes and not the superficial ones. The value of conventional care is not underestimated, but rather bestowed with a more integrated dimension and is now rendered holistic $[1,2]$. According to this approach, the physical and mental manifestations of a disease are now treated as a whole in the medical practice of each specialty. It refers to a more integrated (= holistic) treatment of each morbid process in a human being, who is no longer considered as merely a carbon-based life form (body). Holistic care allows a more thorough consideration of a disease and an escape from traditional mechanistic approaches, which even though have offered a lot in the field of Medicine and Nursing, now seem obsolete. In that context, holistic care, as a set of methods which go beyond the status quo of care, and which are related to treatment methods observed in traditional societies, not only manages to coexist but also to evolve alongside conventional care and medicine. There is an increasing number of scientists who recognize the significant contribution of alternative medicine to the rehabilitation of a patient's health, and also patients who turn to alternative-holistic medicine in their time of need [3].

The purpose of this study was to find current bibliographic data on the value and applications of holistic nursing care. First, the theoretical foundations of holistic care are mentioned, and then, the modern view of the biopsychosocial model of the disease and the holistic approach applications in patient care design are analyzed.

\section{The Holistic Approach to Therapy}

The terms "Holism" and "Holistic" come from “ 'Oגos-holos" a Greek word meaning all, whole, entire, total. Holistic medicine deals with a human being as a whole, i.e. it implements a multifaceted approach to the health-disease issue. Human beings, regarded as whole entities, are the focus of interest and not the individual diseases. Holistic medicine is not a different medical or treatment method, but rather a different philosophy on how to approach health. Symptoms themselves are now considered as the manifestation of a disease. The holistic approach takes into consideration an individual's psychological, sociologi- 
cal and mental views and needs. Each individual consists of body, mind and soul as a unified total and is not just the sum of his body parts. Change in any aspect of an individual's life brings change to every aspect of his existence and differentiates the quality of his whole [4]. The theoretical foundation of nursing itself is intrinsically holistic, since from early on it had been made clear that patient care cannot and should not be one-dimensional. Florence Nightingale herself encouraged holistic care, by recognizing the importance of environment, touch, light, scents, music and silent reflection in the therapy process.

The holistic approach summarizes the psychosomatic approach of a disease and expands it by putting the patient in a specific time and cultural context. It deals with his own special needs and quality of life, by respecting his convictions [5].

The holistic approach has two important aspects. Firstly, it treats each individual as a separate entity, both in biological and social terms. Secondly, it is multidimensional, introducing a less simplistic view on health and disease. These two aspects affect the nurse-patient interaction, as well as the formation of the related research questions. Being able to differentiate between an "objective" and a "subjective" disease is important for a nurse in terms of approaching the patient, interpreting health in various people, situations and environments, and determining his/her actions in order to promote health and prevent diseases. Both the patient and the nurse are considered as subjects. This means that they are both aware of what is important and relative to the case in hand, and that they both have capabilities, alternatives, rights and freedom of choice. Therefore, the main characteristic of their relationship is equality $[1,6,7]$.

The holistic approach is governed by certain principles, which can be summarized in the following:

Each individual is capable of improving his knowledge and skills and changing his behavior towards himself and others.

People are naturally inclined towards being healthy, but are also responsible for their good health status, recovery and taking care of themselves.

A "person" belongs to himself. Therefore, his decisions and developments rightfully belong to him, the "owner".

The main focus of the recovery efforts is the individual and not the disease or injury.

The relationship between health care professionals and their patients should be one of mutual collaboration. People who provide health care intervene on behalf of the adult patient only when he asks for their help or when his health needs cannot be fully satisfied $[4,5]$.

\section{Holistic Nursing Care Theories}

Holistic nursing care provision was based on various theories, each focusing on specific aspects of the relationship between the person with his outside and inside world and his nurse, by respectively differentiating the concept of health. Their common ground, however, is dealing with the person as a whole being. From Maslow to modern holistic theories, the holistic approach embraces the total of human needs and puts them in a hierarchy by accepting a person's individuality and the gravity he puts on each individual need.

\subsection{The Intersystem Model}

The nursing theories of interactive processes are founded philosophically on integrative process models. The latter are based on the so called humanistic philosophy. Based on that, every person is a uniform being which constitutes an energy field in constant interaction with the energy field of the universe. The supporters of such nursing theories deal with a person as a holistic being, which interacts and adapts to the situations that he faces. They support systems theory and suggest that a person is in constant interaction with his environment. In that context, health is a value which ranges constantly from the highest possible well-being to disease. This view entails that people with chronic conditions may lead a healthy life and be satisfied by it, despite their condition $[6,7]$.

\subsection{Callista Roy's Adaptation Model}

The theoretical outline of adaptation in Roy's nursing theory includes a set of hypotheses which can be summarized in the following: A person is a biopsychosocial being in constant interaction with an ever-changing environment. In order for the person to face that ever-changing world, he would have to use biological, psychological and social mechanisms. Adaptation is accentuated as a key concept to a person's positive reaction to environmental changes. A person's adaptation level, which may lead to a positive reaction, includes a zone, indicative of the stimulus range, which disturbs the balances and calls for a new adaptation. It is also called "adaptation" theory, based on "systems" theory, in Helson's adaptation level theory and in humanistic values. It suggests that a system is a set of units interrelated or interlinked together, in order to form a unity or a set. It holds that all systems have input and output data, as well as control and feedback processes, by considering, at the same time, living systems as more complex than mechanical systems. According to Roy's model, human behavior represents an adaptation to environmental and organic forces. The overall resultant of the individual reactions represents the organism's final state. According to Roy, the purpose of Nursing is to aid the person as he adapts to the occurring changes, his biological needs and to his self-perception [8].

\subsection{Theories Based on Integrative Process}

Even though today, care group cooperation and interdisciplinary approach are a given, that was not always the case. Even during the second half of the past century, the person-patient was treated as something different than the object of physicians' and nurses' care. Most health professionals operated with virtually no communication between them, each focusing on their own assignment, with none of 
them being fully aware of the patient and his needs.

\subsection{Rogers's Theory}

That conclusion led Rogers [9] to suggest that a person is a "uniform energy system", which is in "constant reciprocal interaction with the energy system of the universe", and she, thusly, ultimately achieved to drastically affect the nursing field and encourage nurses to deal with a person as a whole unit, both during care design and provision.

According to Rogers, nursing practice aims towards promoting a harmonic interaction between the person and his environment, and reinforcing the cohesion and wholeness of a person's energy field. Its goal is to appropriately guide the organization and synchronization of a person's fields and environment in order to achieve the highest possible health potential.

\subsection{Newman's Health Care System Model}

Newman proposed a health care system model, according to which a person is treated as a whole system with individual interlinked parts and subparts. Stress factors take their toll on people, who have, however, flexible resistance limits and back-ups, which help them defend themselves against these stress factors. According to this model, nursing is directed towards recognizing a person's standards in interaction with the environment, and accepting that interaction as an awareness development process. Disease comes to disrupt the relationships between a person's standards in a more harmonic way [10]. Health refers to a person's ability to interact with his environment and to integrate thinking, feeling and processing of the information contained in all natural systems. Health becomes synonymous with developing awareness. Awareness development and therefore, the route towards health, have to go through new standards realization, transformation and discovery inside a complex living system. "Health" is experienced as a dynamic coexistence of the traditionally viewed health and the traditionally viewed disease. It is a "well-being" information, which reflects the balance state a person's entire being has reached. Nursing is described as a "human experience health care". It actually suggests a health care system model, which considers a person as a whole system with individual interlinked parts and subparts [11,12].

\subsection{Parse's Theory (Human Becoming)}

According to Parse, human is considered as a Being, of his own will, who actively participates inside the world. A person can only be whole when he participates in the cosmic process. He is whole, open and free to choose the ways of the process [13]. Parse had a radical view on health, suggesting that health status is our way of existence inside the world. There is neither a continuum from health to disease, nor a health-disease counterpoint, it is more of the everyday way of existence [13]. This is a new and unique way of viewing health, which provides understanding of the way each person creates his own destiny.
Nursing guides the person to find new ways of existence, to discover meaning in situations, to choose ways of coestablishing his personal health and to live his everyday life [13].

Summarizing the main holistic care theories, it should be noted that final treatment is the overall resultant of many factors related to a patient's "reality", as that is yielded by the individual components, which also include the nurse. The patient, the environment, the nurse and the health objective are all interconnected, revolving around the treatment, which is the main focus.

\section{Bridging the Gap between Theory and Practice}

Despite the repeated efforts to bridge the gap between practical theory, practice and research, little progress has been made in that direction. Besides, the gap does not seem to come from flawed theories, which can, in either case, be evolved and reviewed in order to cover modern practice data, or from deliberate overlook of their calling. Incomplete care provision through health systems, which are not theoretical but totally tangible, is mostly derived by a shortsighted perception of nursing care, which is at best worn out in the context of the biomedical model, and also the lack of resources, which limits interdisciplinary potential and supplementary social care services. Nurses face numerous challenges due mainly to the changes in the health provision system. For example, limiting hospitalization time has limited the pre-operative intervention and programming timeframe. Similarly, insurance company mechanisms have a drastic effect on home care provision, thusly determining to a large extent when and how the nurse takes care of the patients, and the type of services that he/she provides. These changes, despite the fact that they have dealt partially with the health provision cost inflation, have had a negative effect on nursing care provision [14].

However, we must also take into consideration that other issues also need to be resolved, such as education, researchers-clinical nurses interaction, as well as modern nursing practice issues. An important issue is also the lack of theoretical principles from the basic training program. The majority of nurses graduate from secondary education, either public or private, institutions, which focus only on purely practical skills, and give little priority to theoretical foundation and research, thusly rendering the nurses' post-graduation actions limited in consistency, repeatability and precision, and the nurse more of an empiricist and less of a scientist. Holistic care, in particular, requires thorough and careful handling and utilization of all the mental resources of both the patient and his caregiver, who must have some previous training and certain skills $[5,6,15,16]$.

Clinical nurses' further education is equally important to help them deal with certain issues, as is the promotion of the theoretical concepts and principles of holistic care. All clinical nurses should be encouraged to increase their 
knowledge by familiarizing themselves with new theoretical principles and nursing research, through continuing education or basic education programs [16].

\section{Conclusions}

Modern medicine in an attempt to free itself from the rigid, non-scientific views which hindered its development for centuries was led to its over-segmentation in sub-specialties and mechanistic views on human beings. As a result, a disease is treated as a mechanical malfunction of an instrument, rather than as the result of a disturbance in the balance of an organism as a whole and its environment. It drifted apart from observing feelings and the way feelings may be related to the manifestation of a given condition. It does not examine the effect of a person's convictions in his life and the dysfunctions these may cause. It does not take into consideration the great part of interpersonal human relationships in the formation of a disease, while it also degrades mental reality and does not utilize its treating potentials [2,17].

Holistic medicine includes both modern (medical, surgical, etc.) and alternative treatment methods. Natural, mild alternative treatment is applied only when it can guarantee the patient's safe and secure treatment, otherwise it is combined with or replaced by modern medical approaches, with the physician focusing not only on the symptoms and epiphenomena, but also on the patient's overall health condition. Holistic medicine comes to satisfy the health professionals' scientific curiosity, by showing them the versatile potential and benefits of the so called natural or alternative treatment, and that both their patients and their practice could benefit from that $[18,19]$. Human beings are not just biological organisms with mental and emotional manifestations, but a multidimensional entity with a body, mind, soul and spirit. All of these elements interact and affect one another. Health is the state of balance between these elements, which requires the "harmonization" of a person's life with the laws of nature. Health requires self-awareness, personal happiness and self-actualization. The main treatment objective is the person's increasingly conscious and active participation in the race to gain and preserve his health. All available methods are included in the health professional's reservoir, but he has to carefully evaluate and appropriately apply them. The use of these methods by the patients is an indisputable fact, a reality which needs to be taken into serious consideration. If the health professionals are honest when dealing with this reality, then, the patient will also be honest and cooperative. Furthermore, room for more scientific research, which would help demonstrate the above, is required $[15,20]$.

In conclusion, the "technocracy" and depersonalization of conventional medicine, which put off many patients, as well as its failure to treat serious diseases, which reduced its credibility, combined with the fact that some alternative forms of treatment have been recognized as successful by the scientific community are all important reasons as to why holistic care is as widespread as it is today. Holistic care comes to complete the conventional approaches and to promote health to increasingly higher levels of mental and physical well-being and functionality.

\section{References}

[1] Dossey BM. Theory of integral nursing. ANS. Advances in Nursing Science 2008; 31:52-73.

[2] Fassino S. Psychosomatic approach is the new medicine tailored for patient personality with a focus on ethics, economy, and quality. Panminerva Medical 2010; 52:249-64.

[3] Molassiotis A, Fernadez-Ortega P, Pud D, Ozden G, Scott JA, Panteli V, et al. The growth of complementary therapies: and their benefits in the perioperative setting. Journal of perioperative practice 2009 ; 19:382-6.

[4] Erickson HL. Philosophy and theory of holism. Nursing Clinics of North America 2007; 42:139-63.

[5] McEvoy L, Duffy A. Holistic practice--a concept analysis. Nurse Education in Practice 2008; 8:412-9.

[6] Berg GV, Sarvimäki A. A holistic-existential approach to health promotion. Scandinavian Journal of Caring Science 2003; 17:384-91.

[7] Kubsch SM, Hankerson C, Ghoorahoo R. Content analysis of holistic ethics.Complement Therapies in Clinical Practice 2005; 11:51-7.

[8] Roy C. The Roy adaptation model. In Riehl JP. \& Roy C. (Eds.), Conceptual Models for Nursing Practice. Norwalk: Appleton, Century Crofts 1980.

[9] Rogers ME. Nursing science evolves. In: Madrid, M., Barrett, E.A.M., (eds). Roger's scientific art of nursing practice. New York, National League for Nursing Press 1994.

[10] Newman MA. Health as expanding consiousness. National League for Nursing Press. New York, 1999.

[11] Newman MA. Newman's theory of health as praxis. Nursing Science Quarterly 1990a; 3:37-41.

[12] Newman MA. Toward an integrative model of professional practice. Journal of Professional Nursing 1990b; 6:167-173.

[13] Parse, R.R. Man- living - health: A theory of nursing. New York, Wiley 1981.

[14] Streubert HJ, Carpenter DR. Qualitative research in nursing: Advancing the humanistic imperative. Lippincott Williams \& Wilkins, Philadelphia 1999.

[15] Wheeler K. Psychotherapeutic strategies for healing trauma. Perspectives in Psychiatric Care 2007; 43:132-41.

[16] Raya A. Can knowledge be promoted and values ignored? Implications for nursing education. Journal of Advanced Nursing 1980; 15:504-509.

[17] Koslander T, da Silva AB, Roxberg A. Existential and spiritual needs in mental health care: an ethical and holistic perspective. Journal of Holistic Nursing 2009; 27:34-42.

[18] Ledge SD. The duty of nurses to meet patients' spiritual and/or religious needs. British Journal of Nursing 2005; $14: 220-225$. 
[19] McSherry W, Cash K, Ross L. Meaning of spirituality: implications for nursing practice. Journal of Advanced Nursing 2004; 13:934-941
[20] Van Leeuven R, Gusveller B. Nursing competencies for spiritual care. Journal of Advanced Nursing 2004; 48:234-246. 